Published in final edited form as:

JAMA. 2014 May 07; 311(17): 1797-1798. doi:10.1001/jama.2014.967.

\title{
Breastfeeding and Antiepileptic Drugs
}

\author{
Kimford J. Meador, MD \\ Department of Neurology and Neurological Sciences, Stanford Comprehensive Epilepsy Center, \\ Stanford University School of Medicine, Stanford, California.
}

\begin{abstract}
IMPORTANCE-Exposure to antiepileptic drugs during pregnancy is associated with adverse effects on psychomotor development.

OBJECTIVES-To determine whether signs of impaired development appear already during the first months of life in children exposed prenatally to antiepileptic drugs, and to explore potential adverse effects of antiepileptic drug exposure through breastfeeding.
\end{abstract}

DESIGN, SETTING, AND PARTICIPANTS-Mothers at 13 to 17 weeks of pregnancy were recruited in the population-based, prospective Norwegian Mother and Child Cohort Study from 1999 to 2009. The mothers reported on their child's motor and social skills, language, and behavior using items from standardized screening tools at 6 months $(\mathrm{n}=78744), 18$ months $(\mathrm{n}=$ $61351)$, and 36 months $(n=44$ 147) of age. The mothers also provided detailed information on breastfeeding during the first year.

MAIN OUTCOMES AND MEASURES-The risk of adverse development in children according to maternal or paternal epilepsy was estimated as the odds ratio with corresponding $95 \%$ confidence interval, adjusted for maternal age, parity, education, smoking, breastfeeding, depression/anxiety, folate supplementation, and congenital malformation in the child.

RESULTS-At age 6 months, infants of mothers using antiepileptic drugs $(\mathrm{n}=223)$ had a higher risk of impaired fine motor skills compared with the reference group (11.5\% vs $4.8 \%$, respectively; odds ratio $=2.1 ; 95 \% \mathrm{CI}, 1.3-3.2$ ). Use of multiple antiepileptic drugs compared with the reference group was associated with adverse outcome for both fine motor skills $(25.0 \%$ vs $4.8 \%$, respectively; odds ratio $=4.3 ; 95 \%$ CI, $2.0-9.1)$ and social skills $(22.5 \%$ vs $10.2 \%$, respectively; odds ratio $=2.6 ; 95 \% \mathrm{CI}, 1.2-5.5$ ). Continuous breastfeeding in children of women using antiepileptic drugs was associated with less impaired development at ages 6 and 18 months compared with those with no breastfeeding or breastfeeding for less than 6 months. At 36 months,

\footnotetext{
Corresponding Author: Kimford J. Meador, MD, Department of Neurology and Neurological Sciences, Stanford University School of Medicine, 300 Pasteur Dr (Room A343), Stanford, CA 94305-5235 (kmeador@ stanford.edu).

Conflict of Interest Disclosures: The author has completed and submitted the ICMJE Form for Disclosure of Potential Conflicts of Interest. Dr Meador reported serving on the editorial boards of Neurology, Behavior \& Neurology, Epilepsy \& Behavior, Epilepsy.com, and the Journal of Clinical Neurophysiology and on the Professional Advisory Board forthe Epilepsy Foundation; having received travel support from sanofi-aventis; having received research support from GlaxoSmithKline, Eisai, Marinus Pharmaceuticals, Myriad Genetics, NeuroPace, Pfizer, SAM Technology, UCB Pharma, the NIH NINDS: 2RO1-NS38455 (principal investigator), 2U01-NS038455 (multi-principal investigator), 1 R01 NS076665 (consultant), the Patient-Centered Outcomes Research Institute (co-principal investigator), and the Epilepsy Foundation; and having consulted for the Epilepsy Study Consortium that receives money from multiple pharmaceutical companies (in relation to his work for Eisai, NeuroPace, Novartis, Supernus, Upsher Smith Laboratories, UCB Pharma, and Vivus Pharmaceuticals). The funds for consulting forthe Epilepsy Study Consortium were paid to his university
} 
prenatal antiepileptic drug exposure was associated with adverse development regardless of breastfeeding status during the first year. Children of women with epilepsy who did not use antiepileptic drugs and children of fathers with epilepsy had normal development at 6 months.

CONCLUSIONS AND RELEVANCE-Prenatal exposure to antiepileptic drugs was associated with impaired fine motor skills already at age 6 months, especially when the child was exposed to multiple drugs. There were no harmful effects of breastfeeding. Women with epilepsy should be encouraged to breastfeed their children irrespective of antiepileptic drug treatment.

Knowledge of the developmental effects of antiepileptic drugs (AEDs) is inadequate. The first reports of an association of fetal AED exposure with congenital malformations occurred in the 1960s, and the first report of a specific congenital malformation for a specific AED was published in the early 1980s (ie, cleft lip/palate and valproate). Only recently has the scope of differential fetal AED risks exposure for congenital malformations, cognitive deficits, and behavioral disorders begun to emerge.

Concerns over the cognitive and behavioral effects of AEDs exist not only for fetal exposure but have also been raised for exposure via breast milk. Women with epilepsy typically cannot stop AEDs for pregnancy because of the risks from seizures. In this situation, the clinical decision revolves around the relative anatomical and behavioral teratogenic risks of AEDs that are effective for the woman's type of epilepsy. Following birth, additional AED exposure to the infant could be avoided by not breastfeeding. However, risks of breastfeeding when taking AED remain theoretical because of the paucity of supportive research data. In contrast, there are substantial data supporting beneficial effects of breastfeeding for both the child and mother. ${ }^{1}$ Benefits for children who are breastfed include reduced risk of severe lower respiratory tract infections, atopic dermatitis, asthma, acute otitis media, nonspecific gastroenteritis, obesity, type 1 and 2 diabetes, childhood leukemia, sudden infant death syndrome, necrotizing enterocolitis, and possibly positive cognitive effects. Benefits for mothers who breastfeed include reduced risks for type 2 diabetes, breast cancer, ovarian cancer, and maternal postpartum depression. Many AEDs cross into breast milk in measurable concentrations, with some differences between AEDs; however, the long-term neurodevelopmental effects of AED exposure via breast milk remain uncertain.

In the November issue of JAMA Neurology, Veiby et $\mathrm{al}^{2}$ published findings on the developmental outcomes of children who breastfed vs those who did not breastfeed while their mothers were taking AEDs. They recruited women with epilepsy early in pregnancy from the prospective Norwegian Mother and Child Cohort Study (participation rate, 38.5\%) and assessed the children at ages 6 months $(n=223), 18$ months $(n=184)$, and 36 months ( $=139)$, comparing them with a large reference group $(n=77770)$. The main outcome variables were the mothers' ratings of child development and behavior on standardized scales. The most commonly used AEDs were carbamazepine, lamotrigine, and valproate. Investigators found that breastfed children did not differ statistically from nonbreastfed; further, breastfed children tended to have fewer development impairments.

Study strengths included the prospective design, assessing women with and without AED treatment during pregnancy, including fathers with epilepsy, a large reference group, and assessing many potential confounding variables. Weaknesses include self-reported epilepsy 
diagnosis; lack of information on epilepsy type, seizure severity and maternal AED dose/ levels, IQ, and socioeconomic status; lack of AED levels in breastfed children; small AED sub-group sample sizes; unblinded developmental assessments based on maternal ratings; a $60.2 \%$ response rate at age 36 months; and potential for residual confounding.

Only one other previously published study assessed the neurodevelopmental effects of breastfeeding in children whose mothers were taking AEDs. The Neurodevelopmental Effects of Antiepileptic Drugs (NEAD) study is a multicenter prospective investigation that enrolled women with epilepsy early in pregnancy to examine the neuropsychological effects of fetal AED exposure (carbamazepine, lamotrigine, phenytoin, or valproate monotherapy). ${ }^{3}$ Breastfed $(n=84)$ and nonbreastfed $(n=115)$ children were evaluated at age 3 years by assessors blinded to drug and breastfeeding status. The adjusted IQs did not differ between the breastfed group (IQ = 99) and nonbreastfed children (IQ = 98). Thus, 2 prospective wellcontrolled studies have failed to demonstrate any adverse cognitive effects of breastfeeding while taking AEDs.

Some AEDs can induce neuronal apoptosis and dysfunction in surviving neurons of the immature brain, similar to alcohol. ${ }^{4}$ These effects are dose-dependent, require only a single exposure, ${ }^{4}$ and may depend on peak level. The AED levels in breastfed children depend on the amount of AED in the breast milk, amount of breast milk consumed and absorbed, and rate of AED clearance. Data on AED serum levels in the breastfeeding child are sparse, but it is likely that the AED level in the child from breastfeeding is less than the level when exposed in uterofor most AEDs. ${ }^{5}$ Thus, lower AED levels from breast milk exposure may not add to any adverse effects from the higher AED exposure levels in utero.

Veiby etal ${ }^{2}$ also present 6-month outcomes for infants with fetal AED exposure showing higher risk of impaired fine motor skills $(11.5 \%)$ vs a reference group (4.8\%). Veiby et al ${ }^{6}$ previously reported the child outcomes at 18 and 36 months. Children of mothers with a diagnosis of epilepsy but not taking AEDs and children of fathers with epilepsy did not differ from the reference group. Children who had fetal AED exposure compared with the reference group had abnormal scores for gross motor scores ( $7.5 \%$ vs $3.3 \%$ ), sentence skills $(11.2 \%$ vs $4.8 \%)$, and autistic traits $(6.0 \%$ vs $1.5 \%)$ at age 3 years. Subgroups were small, but 3 main AEDs (carbamazepine, lamotrigine, and valproate) exhibited deficits. In contrast, the previously mentioned NEAD study recently reported on age 6-year outcomes of children of mothers with epilepsy taking AEDs (carbamazepine, lamotrigine, phenytoin, and valproate); greater cognitive and behavioral deficits were seen with valproate exposure compared with other commonly used AEDs. ${ }^{7}$ The difference in results may be related to blinded formal cognitive assessments of the children and controlling for maternal IQ in the NEAD study.

Although more data are needed to fully delineate risks and benefits, there are known positive effects of breastfeeding vs a theoretical risk of breastfeeding while taking AEDs. The theoretical risk has not been substantiated by 2 prospective clinical studies. In addition, there are plausible reasons why breastfeeding might not add to any fetal AED adverse effects. Women should be informed of known benefits and potential risks rather than given a blanket statement that breastfeeding while taking AEDs is unsafe. Furthermore, it seems reasonable 
based on available data to recommend breastfeeding in children of women taking AEDs whose children have already been exposed in utero. For women beginning AED after delivery, more caution is advised, especially for AEDs known to be associated with adverse effects on the immature brain.

\section{Acknowledgments}

Funding/Support: This work was supported by a grant from the National Institutes of Health (U01 NS038455).

Role of the Sponsor: The funding source had no role in the preparation, review, or approval of the manuscript.

\section{REFERENCES}

1. Ip S, Chung M, Raman G, Trikalinos TA, Lau JAsummary of the Agency for Healthcare Research and Quality's evidence report on breastfeeding in developed countries. Breastfeed Med. 2009;4(suppl 1):S17-S30. [PubMed: 19827919]

2. Veiby G, Engelsen BA, Gilhus NE. Early child development and exposure to antiepileptic drugs prenatally and through breastfeeding. JAMA Neurol. 2013;70(11):1367-1374. [PubMed: 24061295]

3. Meador KJ, Baker GA, Browning N, et al.; NEAD Study Group. Effects of breastfeeding in children of women taking antiepileptic drugs. Neurology. 2010;75(22):1954-1960. [PubMed: 21106960]

4. Ikonomidou C, Turski L. Antiepileptic drugs and brain development. Epilepsy Res. 2010;88(1):1122. [PubMed: 19825509]

5. Hovinga CA, Pennell PB. Antiepileptic drug therapy in pregnancy II. Int Rev Neurobiol. 2008;83:241-258. [PubMed: 18929086]

6. Veiby G, Daltveit AK, Schjalberg S, et al. Exposure to antiepileptic drugs in utero and child development. Epilepsia. 2013;54(8):1462-1472. [PubMed: 23865818]

7. Meador KJ, Baker GA, Browning N, et al.; NEAD Study Group. Fetal antiepileptic drug exposure and cognitive outcomes at age 6 years (NEAD study). Lancet Neurol. 2013;12(3):244-252. [PubMed: 23352199] 